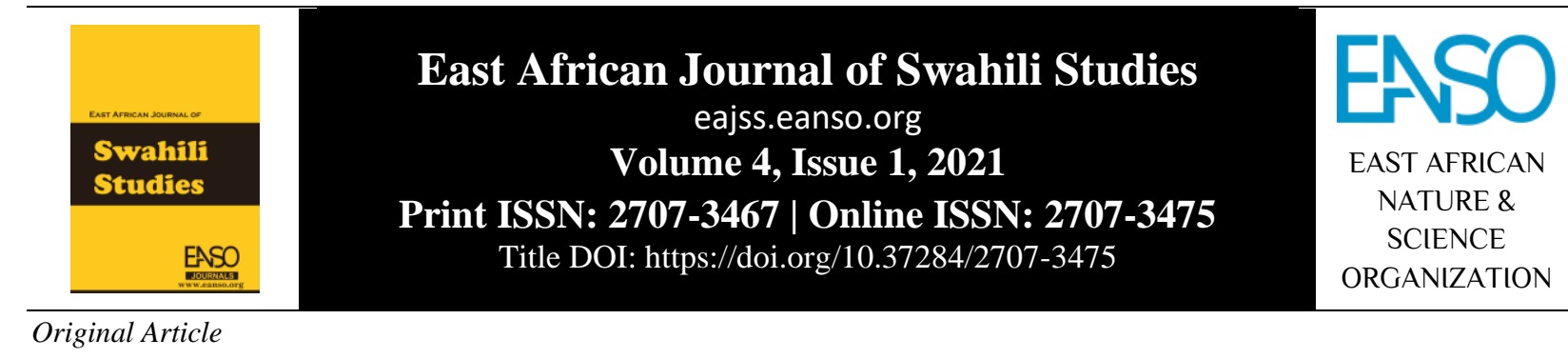

\title{
Nafasi ya Riwaya za Kingano za Kiswahili katika Harakati za Kupigania Hadhi-Msingi ya Mwanamke. Mfano Riwaya ya Marimba ya Majaliwa (2008) ya Edwin Semzaba.
}

\author{
Furaha J. Masatu ${ }^{l}$,Venancia F. Hyera ${ }^{1} \&$ Osmunda R. Ndunguru ${ }^{1 *}$ \\ ${ }^{1}$ Mwenge Catholic University, S.L.P 1226, Moshi-Kilimanjaro, Tanzania. \\ * Barua pepe ya mawasiliano: srdeodathandunguru@gmail.com
}

DOI ya Nakala: https://doi.org/10.37284/eajss.4.1.512

Tarehe ya Uchapishaji: IKISIRI

18 Desemba 2021 Makala haya yanalenga kuonesha namna riwaya za kingano za Kiswahili zinavyoweza kutumika kumpigania na kumkomboa mtu mnyonge hususani

Istilahi Muhimu: mwanamke na mtoto. Baadhi ya watu wanadhani ngano ni kwa ajili ya watoto kuburudika na kuwatia hofu kama njia ya kuwajengea adili. Ukweli ni kuwa,

Ukombozi Wa ngano ni zaidi ya burudani na hofu. Ndani ya ngano mna mambo mazito Mwanamke, yanayoweza kumlea mtoto kwa kumuumba upya ili awe kiumbe kipya cha haki Ufeministi, na usawa duniani bila hofu. Baadhi ya ngano zinaonekana kuvuka mipaka ya Hadhi-Msingi, kitamaduni ya mafunzo anayostahili kupata mtoto. Mfano wa ngano hizo ni Riwaya Za Kingano, Riwaya Ya Marimba

Ya Majaliwa. riwaya ya Marimba ya Majaliwa (2008) ya Edwin Semzaba inayomsawiri mwanamke kama kiumbe mwenye mapaji na uwezo mkubwa hata kumshinda mwanaume dhalimu. Mtazamo huu ni kinyume na mafunzo yanayopatikana katika ngano nyingi tulizonazo sokoni - kutokana na utiisho uliokita katika mfumodume. Makala yanabeba muhtasari wa uchunguzi uliongozwa na nadharia ya Ufeministi kuitalii riwaya ya Marimba ya Majaliwa (2008). Uchunguzi umebaini kuwa, mwandishi amemchora mwanamke kwa mtazamo chanya kwa kuonesha hadhi-msingi alizoumbiwa mwanamke mbali ya changamoto anazozipitia katika kujitetea. Ingawa mwanamke anaonekana kuendelea kukandamizwa na mfumodume, bado ukombozi wa kifikra unamfanya asikubali kuutii na kuuabudu. Katika mapambano haya, mwanamke amefanikiwa kuonesha hadhi-msingi zake tofauti tofauti zikiwemo: huruma na upendo kwa wanyonge, mpinga dhuluma, mjasiri, mwenye bidii, ubunifu, ujuzi, maarifa na nyenzo bora za kumwezesha kuhimili mikikimikiki ya mfumodume. Kwa picha hii, makala haya yatasaidia kuongeza matini za Kiswahili zilizoshughulikia mchango wa riwaya za kingano katika ukombozi wa mwanamke na mtoto. Vilevile, makala haya yatasaidia kuwaongoza

68 This work is licensed under a Creative Commons Attribution 4.0 International License. 
wahakiki na watafiti wanaotarajia kuitalii riwaya ya Marimba ya Majaliwa au kazi nyingine ya kingano ili kumsawiri mwanamke.

\section{APA CITATION}

Masatu, F. J., Hyera, V. F. \& Ndunguru, S. R. (2021). Nafasi ya Riwaya za Kingano za Kiswahili katika Harakati za Kupigania Hadhi-Msingi ya Mwanamke. Mfano Riwaya ya Marimba ya Majaliwa (2008) ya Edwin Semzaba. East African Journal of Swahili Studies, 4(1), 68-81. https://doi.org/10.37284/eajss.4.1.512.

\section{CHICAGO CITATION}

Masatu, Furaha J, Venancia F. Hyera and Osmunda R. Ndunguru. 2021. "Nafasi ya Riwaya za Kingano za Kiswahili katika Harakati za Kupigania Hadhi-Msingi ya Mwanamke. Mfano Riwaya ya Marimba ya Majaliwa (2008) ya Edwin Semzaba". East African Journal of Swahili Studies 4 (1), 68-81. https://doi.org/10.37284/eajss.4.1.512.

\section{HARVARD CITATION}

Masatu, F. J., Hyera, V. F. \& Ndunguru, S. R. (2021) "Nafasi ya Riwaya za Kingano za Kiswahili katika Harakati za Kupigania Hadhi-Msingi ya Mwanamke. Mfano Riwaya ya Marimba ya Majaliwa (2008) ya Edwin Semzaba”, East African Journal of Swahili Studies, 4(1), pp. 68-81. doi: 10.37284/eajss.4.1.512.

\section{IEEE CITATION}

F. J. Masatu., V. F. Hyera, \& O. R. Ndunguru. "Nafasi ya Riwaya za Kingano za Kiswahili katika Harakati za Kupigania HadhiMsingi ya Mwanamke. Mfano Riwaya ya Marimba ya Majaliwa (2008) ya Edwin Semzaba”, EAJSS, vol. 4, no. 1, pp. 68-81, Dec. 2021.

\section{MLA CITATION}

Masatu, Furaha J, Venancia F. Hyera \& Osmunda R. Ndunguru. "Nafasi ya Riwaya za Kingano za Kiswahili katika Harakati za Kupigania Hadhi-Msingi ya Mwanamke. Mfano Riwaya ya Marimba ya Majaliwa (2008) ya Edwin Semzaba". East African Journal of Swahili Studies, Vol. 4, no. 1, Dec. 2021, pp. 68-81 doi:10.37284/eajss.4.1.512.

\section{UTANGULIZI}

Makala haya yanarejelea matokeo ya uchunguzi wa nafasi ya riwaya za kingano za Kiswahili katika harakati za kupigania hadhi-msingi ya mwanamke kwa kuitalii riwaya ya kingano ya Marimba ya Majaliwa (2008) ya Edwin Semzaba. Makala yamegawanywa katika mada ndogo tano. Nazo ni usuli wa mada, nadharia teule, kuhusu riwaya teule, riwaya ya kingano kama jukwaa la kumpigania mwanamke, na hitimisho. Mada ndogo zote zinalenga kuthibitisha namna riwaya za kingano za Kiswahili zinavyotumika kupambana na mfumodume ikiwa ni njia mojawapo ya kitaaluma na kisanaa ya kumlingania mwanamke na mtoto.

\section{Usuli wa Mada}

Jamii nyingi zilizotawaliwa na, au zinazotawaliwa na mfumodume zinaonekana kumchukulia mwanamke kama kiumbe dhaifu anayeweza kutendewa udhalimu bila hofu wala shaka. $\mathrm{Na}$ kwamba, mwanamke anaweza kudhulumiwa na kunyimwa haki zake za msingi mbele ya jamii nzima na asiwepo wa kumtetea mwanamke. Kwa sababu tu, kila mmoja anaona tendo husika ni sahihi (Levine, 1977). Pamoja na dhuluma hizo, bado mwanamke anaendelea kutazamwa kama kiumbe wa kutatua mahitaji ya aina na namna yoyote inayotakiwa na mwanaume mahali na wakati wowote ule. Kwa karne nyingi, mfumo huu umeendelea kumtenga mwanamke katika masuala muhimu yanayoamua hatima ya umiliki, hadhi, heshima na ushiriki wake kiutamaduni, kisiasa, kijamii na kiuchumi. Mfumo huu unatajwa kumnyang'anya mwanamke uwezo wa kujitambua kifikra, kiubunifu na kimaendeleo (Pasque, 2011).

Hali ya dhuluma dhidi ya mwanamke inatajwa kuwa mbaya zaidi katika mataifa yanayoendelea (Hunter na Sellers, 1998). Jamii nyingi za mataifa haya, hususani katika Asia, Mashariki ya kati na Afrika zinaonekana kuwakweza wanaume na kuwadunisha wanawake. Mwanamke bado hana sauti wala nguvu ya maamuzi katika jamii. Ingawa hali inatajwa kuwa mbaya, bado wanaharakati wanaendelea kupambana na mfumo huu usio na tija kwa jamii. Kupitia juhudi hizo, kunaendelea kupatikana mafanikio chanya dhidi ya mfumodume (Pearson, 2000). Mafanikio hayo yanatokana na juhudi kadha wa kadha za kuielimisha jamii ili kutambua nafasi ya mwanamke katika ushiriki sawa na umiliki sawa wa maamuzi na fursa za kitamaduni, kijamii, kisiasa na kiuchumi. Juhudi 
hizo zinapitia katika majukwaa mbalimbali ya kitaaluma, kimahakama, kifasihi, kijamii, kiuchumi na kitamaduni. Elimu ya haki na usawa, elimu ya ujasiriamali, upatikanaji na uwezeshaji wa raslimali za kumuinua mwanamke ni sehemu ya hatua katika juhudi husika. Hatua nyingine ni kutungwa kwa sheria na sera zisizokandamizi kwa mwanamke.

Mengine ni kuwaibua na kuwatambulisha wanawake waliofanikiwa ili wawe chachu kwa jamii zilizoshikilia udhalimu dhidi ya mwanamke. Pia, kuwekwa kwa mazingira rafiki ya kumsaidia mwanamke kujikwamua kielimu, kiuchumi na kiutawala / kiuongozi. Juhudi hizi bado zinakabiliwa na changamoto kadha wa kadha ambazo kiini chake kipo ndani ya mfumodume uliokita mizizi katika jamii nyingi. Hata hivyo, pamoja na changamoto, imebainika kwamba, kadri wakati unavyoendelea ndivyo wanawake nao wanavyozidi kutambua athari ya mfumodume kwao na kwa jamii. Pia, wanazidi kuitambua hali yao duni na kuanza kutafuta njia ya kujinusuru kutoka katika hali hii ili kurudisha hadhi-msingi yao iliyopokwa kwa karne nyingi (Pearson, 2000). Jukwaa la kisanaa kama moja ya majukwaa tumizi katika harakati za kumpigania mwanamke, halijabaki nyuma katika juhudi za kumuinua na kumpa hadhi stahiki mwanamke. Jukwaa mojawapo la kisanaa ni fasihi andishi (Masatu na wenzake, 2020). Miongoni mwa kazi za fasihi andishi zinazoonekana kuonesha mafanikio katika kuibua hadhi-msingi ya mwanamke na kuusuta mfumodume unaomdunisha mwanamke ni riwaya ya Marimba ya Majaliwa (2008) ya Edwin Semzaba. Riwaya ya Marimba ya Majaliwa ni miongoni mwa riwaya za kingano zilizotungwa kwa minajili ya kusomwa na watoto (rejea dibaji ya Marimba ya Majaliwa, uk. iv - vi).

Riwaya za kingano ni masimulizi bunilizi andishi ya kinathari yanayobeba sifa za ngano. Mulokozi (2017) anafasili ngano kama hadithi ya kimapokeo itumiayo wahusika kama wanyama, mazimwi, miti na watu kuelezea au kuonya kuhusu maisha. Mulokozi anaongeza kuwa, kwa kawaida, ngano husimuliwa na wazazi, walezi au mabibi na mababu kwa watoto au wajukuu wao kwa lengo la kuwapa mafunzo muhimu ya maisha, na wakati huo huo kuwaburudisha. Kwa asili, ngano ni hadithi chini ya fasihi simulizi. Lakini si ajabu kwa zama hizi kukutana na ngano katika fasihi andishi. Tena zikiwa siyo sehemu ya hadithi fupi bali hata kuwa riwaya ama tamthilia au ushairi.

Baadhi ya wasomaji wanaiona riwaya hii kuwa ni ngano ya kuburudisha zaidi wala hamna mambo mazito kama zilivyo riwaya dhati. Uchunguzi wetu katika riwaya ya kingano ya Marimba ya Majaliwa (2008) imethibitisha kwamba mtazamo wa watu hawa siyo sahihi. Mwandishi wa riwaya hii ametumia kalamu na karatasi yake kuanza kumfunda mtoto kujua na kuchukia uovu unaotendwa katika jamii kiasi cha kuathiri ustawi wa maisha, haki na usawa baina ya mwanamke, mwanaume na mtoto kwa sasa na siku za usoni. Mwandishi anaonesha kuwa, mateso anayopitia mtoto kwa sababu ya udhalimu wa mfumodume, daima humgusa mwanamke moja kwa moja; na kinyume chake.

\section{NADHARIA YA UFEMINISTI}

Ufeministi ni nadharia inayohusu ukombozi wa mwanamke katika nyanja zote za maisha kiuchumi, kiutamaduni, kijamii, kidini na kisiasa. Hii ni nadharia inayopinga mfumodume (Maggie, 1996). Historia ya nadharia ya Ufeministi ni tofauti kidogo na nadharia nyingine nyingi. Utofauti wake unatokana na ukweli kwamba, awali dunia haikuwapa nafasi wanawake kupata na kutumia fursa za kujikwamua nyanjani kwa namna sawa na wanaume. Ndiyo maana, unapotafuta mwasisi mahususi hasa wa nadharia hii na mwaka mahususi hasa wa kuanza kwake unakumbana na rekodi tofauti tofauti zenye kuwatambulisha wanawake kadhaa kutoka mataifa na kada tofauti tofauti wakitajwa kuwa waasisi. Hali hii ni tofauti na nadharia za awali zilizoasisiwa na wanaume. Kwa hiyo, hata historia ya nadharia ya Ufeministi nayo ni uthibitisho wa athari za mfumodume duniani kote. Aidha, dhana ya ufeministi haiwezi kuelezwa kwa namna moja na rahisi kutokana na namna ilivyoanza kihistoria lakini pia tofauti za kimapokeo zinazoishi duniani.

Mambo yanayochangia ugumu huu ni pamoja na: watu kulinda asili ya vizazi vyao, kulinda utambulisho wa mbari (koo/makabila), mapokeo ya kijinsia, matabaka ya kijamii, utaifa na wingi wa tambulisho za kila aina zinazojitokeza kwa mtu mmoja (Pasque, 2011). Kwa mfano, mwanamke kama ilivyo kwa mwanaume, anao utambulisho 
mwingi unaoibua changamoto pindi unapomhusisha katika kuujadili Ufeministi. Kwa sababu, yeye ni mama, dada, bibi, shangazi, mke, mke mwenza, mkwe, mfanyakazi; anatoka katika mbari fulani ya kizazi cha fulani, bara fulani, familia yenye hadhi fulani; kiongozi katika jamii, kanisani na msikitini. Vilevile, Ufeministi wenyewe unaonekana kubadilikabadilika kutokana na ukweli kwamba, kila zama huibua ajenda mpya inayokimbizana na wakati.

Pamoja na changamoto hizi, bado wanataaluma wana kitu cha kusema juu ya Ufeministi. Mfano, Hewitt (2010) anawatambua baadhi ya wanawake kama sehemu ya wanaharakati wa mwanzo waliochangia kuzaliwa kwa Ufeministi. Wanawake hao ni Elizabeth Candy Stanton na Mitilda Joslyn Gage. Pia, Hewitt (2010) anauona Ufeministi kama jukwaa la kisiasa na kijamii lililoanza mwishoni mwa karne ya 18 na kushika kasi kiasi katika karne ya 19 katikati. Hata hivyo, Pasque (2011) anatujuza kwamba, ingawa mwanzo wa nadharia hii una kiwingu, hatuna budi kutambua kuwa, Ufeministi kama jukwaa la kitaaluma na kisanaa uliimarika mno katika karne ya 20 mwishoni.

Hooks (2000) amewahi kusema kwamba, Ufeministi ni harakati za kukomesha ubaguzi wa kijinsia, unyonyaji wa kijinsia, na ukandamizaji wa kijinsia. Kwa kifupi tunaweza kusema kuwa, Ufeministi unajihusisha na ukombozi wa dhati dhidi ya udhalimu wa kijinsia kwa jamii nyonge ya kike. Harakati za kumkomboa mwanamke zinatambua kuwapo kwa udhalimu uliojichimbia mizizi katika kani kuu tatu; ambazo ni: kani ya mtu binafsi, kani ya kitaasisi na kani ya kijamii au kiutamaduni (Hardiman, Jackson \& Griffin, 2010). Kani ya mtu binafsi inahusiana na tabia na matendo ya mtu yaliyokita katika mapokeo ya kijamii dhidi ya udhalimu kwa mwanamke. Kani ya kitaasisi inagusa sera, sheria, kanuni, desturi na mila zinazotungwa na chama, shirika na taasisi za kijamii kisha kugeuka sheria za kuwadunisha baadhi ya wanajamii huku zikiwanufaisha wengine kwa misingi ya jinsi zao. Taasisi hizi ni pamoja na dini, serikali, elimu, sheria, vyombo vya habari na mfumo wa afya. Kani ya tatu inahusisha zaidi mambo ya kijamii hususani desturi, imani za dini, wajibu wa kijamii, lugha, muziki, na sanaa ambayo hutumika kujenga na kuimarisha imani kuwa kundi maslahi moja (wanaume) ni bora kuliko jingine (wanawake na watoto).

Kutoka katika kani hizi tatu, ndipo tunapopata misingi mitatu mahususi ya Ufeministi. Msingi wa kwanza unaaminisha kuwa, wanawake wanacho kitu cha thamani kinachochangia katika kila uga wa maisha duniani kote. Msingi wa pili unasisitiza kwamba, wanawake kama kundi lililokandamizwa, wamejikuta hawawezi kuthibitisha uwezo wao, kuthaminika na kuaminika, au kuwa na ushiriki kamili katika jamii. Msingi wa tatu ni ule unaohamasisha harakati, tafiti na maandiko ya kifeministi kutokujifungia katika kuhakiki au kukosoa peke yake, bali yaende mbali zaidi kwa kuleta mageuzi ya kijamii. Mageuzi hayo ni sharti yatokee katika kani zote tatu ili ukombozi wa kweli uonekane kwa mtu mmoja mmoja na kwa jamii nzima katika nyanja zote za maisha.

Kani na misingi hii ndiyo inayotupatia hadhi-msingi ya mwanamke. Tunapochunguza maandishi kwa jicho la Ufeministi hatuna budi kutambua kwamba, mwanamke kama alivyo mwanaume ana hadhi yake kwa asili aliyoumbiwa na Mungu. Hadhi asili ya mwanamke ndiyo tunayoitambulisha katika andiko hili kama hadhi-msingi. Hadhi-msingi ya mwanamke imo katika uwezo wake wa asili aliozaliwa nao unaoifanya dunia iendelee kuwapo. Hadhi-msingi ya mwanamke imo katika utu, busara, subira, upambanaji, ujasiri, ubunifu, uzazi, ulezi na upendo. Pia, hadhi-msingi imo katika haki, usawa, utetezi wa wanyonge, kuchukia uovu, bidii, maarifa, stadi, uvumbuzi, uadilifu, faraja, hamasa, ualimu na uongozi (Hardiman, Jackson \& Griffin, 2010). Kwa muda mrefu, hadhi-msingi ya mwanamke imekuwa ikifunikwa na mfumodume. Kwa hiyo, mtu yeyote anayekusudia kutumia jukwaa la Ufeministi kuanzisha au kuendeleza ajenda ya ukombozi endelevu wa kijinsia ni lazima azitambue kani tatu, malengo makuu ya Ufeministi na hadhi-msingi ya mwanamke (Pasque, 2011).

\section{KUHUSU RIWAYA YA MARIMBA YA MAJALIWA}

Marimba ya Majaliwa ni riwaya ya kingano ya Kiswahili. Riwaya hii ilitungwa na mwandishi nguli wa riwaya na tamthilia za Kiswahili nchini Tanzania anayeitwa Edwin Semzaba na kuchapishwa mnamo mwaka 2008. Utunzi wa 
riwaya hii ulitokana na msukumo wa mwandishi kutaka kukidhi mahitaji ya shindano la hadithi za Kiswahili za watoto lililofadhiliwa na nchi ya Swideni kupitia shirika lake la kimataifa la maendeleo lijulikanalo kama SIDA ${ }^{1}$. Riwaya hii iliibuka kidedea na kuchapishwa na kampuni ya $\mathrm{E}$ \& D Vision Publishing chini ya uratibu wa Mradi wa Vitabu vya Watoto Tanzania, mfadhili akiwa ni SIDA.

Riwaya hii ina jumla ya sura 60. Sura ya kwanza inazungumzia shindano la upigaji marimba huko Zanzibar ambako Majaliwa aliibuka kidedea. Pia, sura inatoa ratiba ya mashindano ya marimba na kupotea kwa marimba ya Majaliwa katika mazingira ya kutatanisha. Sura ya pili inahusu mwanzo wa safari ya Majaliwa katika kuitafuta marimba yake. Sura ya tatu inahusu changamoto mbalimbali alizokabiliana nazo Majaliwa katika harakati za kuelekea Zanzibar kwa mara nyingine ili kuitafuta marimba yake. Sura ya nne anaonekana bibi anamkabidhi na kumwelekeza Majaliwa jinsi ya kutumia jiwe la rubi punde anapohisi njaa. Sura ya tano, bibi anamweleza Majaliwa chanzo cha babu yake kumchukia na mahali ilikopatikana marimba nzuri ya nyuzi ishirini. Sura ya sita, Majaliwa anatumia rubi kwa mara ya kwanza bila msaada wa bibi yake ili kupata kifungua kinywa. Sura ya saba inamuonesha Majaliwa akiwa hatarini katika harakati za kutafuta marimba - akielekea Tanga. Sura ya nane inaelezea Majaliwa akiwa ndani ya tumbo la nyangumi.

Sura ya tisa rubi kutokufanya kazi kutokana na Majaliwa kula chakula kupitia kinywani. Sura ya kumi inahusu safari ya Majaliwa mkoani Tanga na masimulizi ya umaarufu wa mkoa wa Tanga. Sura ya kumi na moja inahusu msaada wa Majaliwa kwa kijana mchunga mbuzi wilayani Handeni mkoani Tanga. Sura ya kumi na mbili inahusu msisitizo wa bibi kwa Majaliwa juu ya umuhimu wa kuwa mtii. Sura ya kumi na tatu inahusu safari ya bibi na Majaliwa ndani ya mapango ya Amboni mkoani Tanga. Sura ya kumi na nne inahusu safari ya bibi na Majaliwa katika vilele vya Mlima Kilimanjaro. Sura ya kumi na tano inahusu Majaliwa kujikuta katika mikono ya askari wa geti la Mlima

\footnotetext{
${ }^{1}$ SIDA - The Swedish International Development Cooperation Agency (https://www.sida.se/en)
}

Kilimanjaro. Sura ya kumi na sita inahusu harakati za Majaliwa kuendelea kumfuatilia Kongoti wilayani Moshi, mkoa wa Kilimanjaro. Sura ya kumi na saba inahusu Majaliwa kunusurika kifo baada ya dereva teksi kutaka kumuua Majaliwa, wilayani Moshi - Kilimanjaro. Sura ya kumi na nane na kumi na tisa zinahusu matembezi ya bibi na Majaliwa katika sehemu mbalimbali za mkoa wa Arusha.

Sura ya ishirini inahusu Majaliwa kukumbana na mwizi kijana ndani ya soko mkoani Arusha. Sura ya ishirini na moja inahusu Majaliwa kufanikiwa kulitoka kundi la watu wenye hasira kali mkoani Arusha. Sura ya ishirini na mbili inahusu simulizi ya bibi kwa Majaliwa kuhusu mtu aliyeitwa Lodwaa wa kijiji cha Loiteki. Sura ya ishirini na tatu inahusu Majaliwa kujikuta katika himaya ya Simba mkoani Manyara. Sura ya ishirini na nne ni safari ya Majaliwa kuisaka marimba yake mkoani Singida. Sura ya ishirini na tano inahusu matembezi ya bibi na Majaliwa katika mapango ya Kiomboi mkoani Singida. Sura ya ishirini na sita inahusu kuahirishwa kwa shindano la marimba mkoani Singida na kutolewa kwa ratiba ya shindano la marimba mkoani Tabora. Sura ya ishirini na saba inahusu Kongoti kujikuta hatarini kwa kumwagikiwa na sufuria la kitoweo cha kuku wa moto wakati anapanga kumsukuma Majaliwa. Sura ya ishirini na nane inahusu mizunguko ya bibi na Majaliwa mkoani Tabora na vitongoji vyake na hatimaye kuingia mkoani Shinyanga.

Sura ya ishirini na tisa, bibi anamsimulia Majaliwa uumbaji wa Mungu kwa viumbe. Sura ya thelathini inahusu harakati za Majaliwa kutafuta tangazo la shindano la marimba akiwa stendi mkoani Shinyanga. Sura ya thelathini na moja inahusu Majaliwa kupata kibarua ndani ya mgodi wa Mwadui mkoani Shinyanga na kuwasili kwake mkoani Mwanza. Sura ya thelathini na mbili inaonesha safari ya bibi na Majaliwa kutoka Mwanza kuelekea Butiama - Musoma kisha kurudi Mwanza. Sura ya thelathini na tatu inaonesha Majaliwa kumfuata Kongoti hospitalini Bugando mkoani Mwanza. Sura ya thelathini na nne inahusu Majaliwa na Kongoti kuondoka Mwanza kuelekea 
Bukoba mkoani Kagera kwa usafiri wa meli. Sura ya thelathini na tano inahusu kuanguka kwa marimba feki ndani ya maji na ujio wa bibi melini usiku - kisha kulitalii Ziwa Viktoria. Sura ya thelathini na sita inahusu meli kutia nanga mjini Bukoba, utalii wa mji na bibi kumwelezea Majaliwa maana ya Kaitaba baada ya kupita katika uwanja wa mpira wa Kaitaba mjini Bukoba.

Sura ya thelathini na saba inahusu kutolewa kwa ratiba ya mashindano ya marimba yaliyopangwa kufanyika katika uwanja wa Kaitaba. Sura ya thelathini na nane inahusu safari ya kuelekea Kigoma kwa basi na kitendo cha majambazi kulivamia basi kisha polisi kuokoa mali, maisha yao na maisha ya abiria. Sura ya thelathini na tisa inahusu harakati za Majaliwa kuyasaka marimba yake mkoani Kigoma. Sura ya arobaini inahusu Majaliwa kutalii katika maeneo ya Ujiji na hifadhi ya Taifa ya Sokwe ya Gombe. Sura ya arobaini na moja inahusu Majaliwa kupata nafasi ya kutumbuiza kabla ya ufunguzi wa shindano ndani ya ukumbi wa Hoteli ya Lake Tanganyika mkoani Kigoma. Sura ya arobaini na mbili inahusu Majaliwa kuwindwa na kumkwepa Kongoti katika Hoteli ya Lake Tanganyika kwa kuingia hoteli nyingine.

Sura ya arobaini na tatu inahusu ujio wa bibi kwa Majaliwa na simulizi yake juu ya mtu aliyekosa utii. Sura ya arobaini na nne inahusu Majaliwa kuwasili Jijini Mbeya na kupata kibarua Kileleni Lodge. Sura ya arobaini na tano inahusu Majaliwa kufanikiwa kuyachukua Marimba kutoka kwa Kongoti ila hayakuwa marimba halisi anayoyatafuta. Sura ya arobaini na sita inahusu historia ya Mtemi Mkwawa. Sura ya arobaini na saba inahusu safari ya Majaliwa kwenda kuangalia mashindano ya mashairi Kalenga, mkoani Iringa. Sura ya arobaini na nane inahusu ushiriki wa Majaliwa katika shindano la utambaji wa mashairi huko Kalenga, mkoani Iringa. Sura ya arobaini na tisa inahusu bibi na Majaliwa ndani ya Hifadhi ya Taifa ya Selous. Sura ya hamsini inahusu safari ya bibi na Majaliwa kupitia maeneo kadhaa ya nchi. Mathalani Bonde la Mto Rufiji, Mahenge, Njombe, Ziwa Nyasa, Tunduma, Mbeya hadi Songea. Sura ya hamsini na moja inahusu historia ya Vita vya Majimaji chini ya nabii Kinjekitile. Sura ya hamsini na mbili inahusu safari ya bibi na Majaliwa kutoka Songea kuelekea mkoani Lindi eneo la Kilwa Kivinje.
Sura ya hamsini na tatu inahusu shindano la marimba katika mikoa ya Ruvuma, Mtwara na Lindi kisha kuingia kwao (bibi na Majaliwa) na kutalii maeneo mbalimbali ya Jiji la Dar es Salaam. Sura ya hamsini na nne inahusu safari ya Majaliwa kutoka mkoani Dar es Salaam kuelekea mkoani Morogoro. Sura ya hamsini na tano inahusu Majaliwa kuwakwepa Jurango na wenzake mjini Morogoro. Sura ya hamsini na sita inahusu safari ya Majaliwa kuelekea Dodoma na kuyapata marimba yake halisi yaliyoibiwa na Kongoti. Sura ya hamsini na saba inahusu changamoto mbalimbali zinazomkabili Majaliwa akiwa njiani kuelekea Dodoma. Sura ya hamsini na nane inahusu Majaliwa kuvuka mto kwa kutumia kamba baada ya daraja kusombwa na maji, pamoja na historia ya Mtemi wa Ugogo. Sura ya hamsini na tisa inahusu kuwasili kwa Majaliwa na historia ya mji wa Dodoma kuwa makao makuu ya nchi ya Tanzania. Sura ya sitini inahusu ushindi rasmi wa Majaliwa na hitimisho la shindano la marimba kitaifa, mkoani Dodoma.

Kwa jumla, sura zote 60 zinahusu vita dhidi ya dhuluma, usaliti, chuki, mateso na manyanyaso kwa watu wanyonge wasio na sauti za kujitetea katika jamii. Wanyonge hawa ni mwanamke na mtoto. Lugha iliyotumika ni ya Kiswahili sanifu iliyosheheni wingi wa semi zinazoendana na kundi lengwa, yaani watoto. Aidha, zipo taswira muhimu kama vile: rubi, bibi, babu, jeshi la vibwengo na marimba. Wahusika wake ni wa kubuni na halisi. Wahusika waliosafiri sana na mwandishi ni Majaliwa, bibi na Kongoti. Mandhari na mazingira teule ni halisi zaidi yanayopatikana nchini Tanzania, japo yapo ya kufikirika pia. Msuko wake ni wa motifu ya safari kwa kuitalii Tanzania Bara na Tanzania Visiwani (Zanzibar). Maeneo yaliyotumika kuijenga riwaya hii ni: Mafia, Unguja, Tanga, Moshi, Arusha, Iringa, Mwanza, Kigoma, Dodoma, Bukoba, Morogoro, Singida, Tabora, Butiama, Shinyanga, Songea, Lindi, Mtwara, Tunduru, na miji mingine mingi ya Tanzania.

Mbali na msako wa marimba, mwandishi ametumia kalamu yake kutambulisha na kutangaza maeneo mengi ya Tanzania yenye kubeba historia na vivutio vya utalii vilivyo adimu na adhimu zaidi hapa duniani. Maeneo hayo ni pamoja na: Mapango ya Amboni Tanga (uk. 52), Mlima Kilimanjaro (uk. 60), Hifadhi ya Taifa ya Arusha, Bwawa la Nyumba 
ya Mungu, Hifadhi ya Wanyama Tarangire, Mlima Meru, Ziwa Eyas na Ziwa Natron (uk. 74 - 75). Pia, Michoro ya Kale huko Kondoa Irangi (uk. 104), Hifadhi ya Wanyama ya Serengeti (uk. 130), Hifadhi ya Sokwe Gombe (uk. 156), Kimondo cha Mbozi (uk. 165), Hifadhi ya Selous (uk. 190), kutaja kwa uchache.

\section{Riwaya ya Kingano kama Jukwaa la Kupigania Hadhi-Msingi ya Mwanamke}

Riwaya ni mojawapo ya majukwaa yanayotumika katika harakati za kumkomboa mwanamke. Riwaya ya kingano ya Marimba ya Majaliwa ni mfano mzuri wa majukwaa hayo. Kupitia mifano mbalimbali kutoka riwaya hii teule, ukweli wa hoja ya riwaya ya kingano kutumika kumpigania mwanamke umethibitika. Mifano hii ni nukuu kutoka katika riwaya teule ambapo kila nukuu imebeba hoja kuu moja inayotambulishwa kupitia mada ndogo nne (4). Aidha, ndani ya kila hoja mna vyanzo vya maarifa na taarifa vilivyorejelewa ili kuzishadadiza hoja husika.

\section{Mwanamke Kiumbe Mpambanaji Aliyejaa Huruma, Utu na Ari ya Kutetea Wanyonge}

Mpambanaji ni mtu hodari, shujaa au anayejaribu kufanya jambo pasipokuwa na hofu ya kupambana na kukabiliana na changamoto katika nyanja mbalimbali za maisha ili kutimiza malengo yake (TUKI, 2005). Maisha ya mwanadamu aghalabu mtu mnyonge ni mapambano kamili katika kusaka usawa. Kila mtu hana budi kupambana. Lakini mapambano yasiyojali huruma, utu, upendo na ari njema huishia kuleta machungu badala ya wema. Wakati mfumodume ukipambana kuminya hadhimsingi hizi, mwanamke aliyejikomboa anazichomoza kama njia ya kusaidia kuviondoa vigingi vinavyominya upatikanaji wa haki na usawa.

Mwandishi Edwin Semzaba amemtumia mhusika bibi kuonesha hadhi-msingi aliyonayo mwanamke. Bibi amechorwa kama mwanamke mwenye utu, uvumilivu, ari, huruma na upendo kwa wanyonge. Bibi anawakilisha kundi la watu wanyonge hususani wanawake waliojitambua kiasi cha kuwa tayari kufa kwa ajili ya kupambana na mfumodume - kwa hali na mali ili kuleta haki na usawa kwa jamii. Katika kulidhihirisha hilo, mwandishi anasema;
Majaliwa hakubanduka pale ingawa ilikuwa tayari usiku wa manane. Aliegemea kichwa chake akiwaza. Mara akaguswa na mtu begani. Akaruka kwa hasira akifikiri ni Kongoti amekuja waendelee kupigana. "Niachie" akasema kwa ukali. Alipomwangalia aliyemgusa akakuta ni bibi yake. "Mbona mkali?" "Mbona kama unalia?" Bibi yake aliuliza. "Marimba imetumbukia ziwani." Majaliwa alimwambia bibi yake akionesha nyuma ya meli kwa kidole. "Itakuwa tumeiacha kama kilomita tano kutoka hapa." "Ilikuwaje?" Bibi aliuliza taratibu. "Kongoti..." Majaliwa alianza kuelezea lakini bibi alimkatisha. "Hakujaharibika kitu mjukuu wangu." Palepale bibi aligeuka tai mkubwa na kuruka kuelekea meli ilikotokea. [...]. [...] Alipotua tu aligeuka kuwa mtu, marimba ameyashika mkononi. "Nimerudi mjukuu wangu" bibi akasema. "Asante bibi." "Umenisumbua bure mjukuu wangu," bibi alisema huku akimpa yale marimba. "Haya marimba siyo yenyewe. Nilijua tangu mwanzo kuwa siyo yenyewe. Lakini nilitaka nisikuudhi" (uk. 140 - 142).

Nukuu hii ni miongoni mwa mifano inayoonesha utu, huruma, subira na juhudi zisizo na ukomo za bibi katika kumtetea mtu mnyonge. Jitihada za bibi hazikuishia kwenye kujitokeza na kutekeleza maelekezo ya mtu mnyonge pekee. Pia, alijitahidi kumpa mjukuu wake hamasa, mbinu mbalimbali, nguvu za ziada na elimu ya utambuzi ili aweze kuyapata marimba yake halisi na hatimaye kuibuka mshindi wa shindano la kitaifa la upigaji wa marimba. Mwandishi amebainisha kuwa mwanamke aliyejikomboa kifikra na kimaarifa ana nafasi kubwa katika kuvikabili vihunzi vinavyozuia kufikiwa kwa mafanikio na ustawi endelevu wa jamii.

Tunaona namna mwanamke bibi alivyopambana mchana na usiku, juu na chini, majini na nchi kavu mradi tu lengo liweze kukamilika. Bibi anaonekana kupambana na kundi la wanaume waliolindwa na kuwezeshwa na Babu - kisilaha na kimbinu. Zaidi sana, bibi alipambana na mwanaume mnyonge lakini jeuri kutokana na mfumodume uliomlea na kumkuza. Huyu si mwingine bali Majaliwa ambaye mara kwa mara alikuwa akikiuka miiko na wakati mwingine kushinikiza kutenda mambo ambayo yangemuondoa kwenye rada sahihi ya mapambano 
(uk 45 - 49). Katika mapambano yote haya - kutoka kundi la babu na mwanaume mnyonge Majaliwa, tunaona bibi hakukata tamaa wala kurudi nyuma.

Huu ni uthibitisho kwamba, mwanamke kwa asili ni kiumbe mwenye uwezo wa kupambana bila kuchoka katika hali zote mradi tu apate ari na utashi wa kufanya hivyo. Kwa mujibu wa Rutherford, Marecek na Sheese (2012) na Masatu na wenzake (2020), mwanamke ameumbwa kwa namna ambayo akidhamiria kulipata jambo, huwa harudi nyuma wala kuogopa hatari yoyote inayomkabili au itakayomkabili. Kwa sababu, hukinai hali ya awali na kutokuihitaji tena. Mwanamke aliyefikia hatua hii, hugeuka mpambanaji na jasiri kama simba mawindoni.

Kwa mantiki hii, kumbe kwa asili mwanamke siyo kiumbe duni bali mfumodume katika jamii ndio unaomdunisha kiasi cha yeye kujikuta pasipo kujitambua akiishia kukubaliana na unyonge huo. Kwa hiyo basi, ukombozi wa kifikra unahitajika ili kuchochea uwezo na hasira za asili za mwanamke ili kupambania haki na usawa kwa faida endelevu ya jamii na mtu mmoja mmoja. Kwa kutumia nadharia ya Ufeministi, tunahitimisha kwamba huu ni ushahidi kuwa, riwaya za kingano zinaweza kutumika kumfunda mtoto wa jinsi yoyote kujua ukweli wa kihistoria na ubaya wa mfumodume na namna ya kuusambaratisha.

\section{Mwanamke Kiumbe Jasiri Asiyekata Tamaa Kirahisi}

Jasiri ni mtu asiyekuwa na hofu, na mwenye hali ya kuthubutu kufanya maamuzi bila kujali hatari zitakazompata (Ward, 2001). Ujasiri ni kujitoa mhanga hata kufa ikibidi mradi tu lengo lifanikiwe kwa asilimia mia au kwa kiasi fulani. Moja ya sifa ya mtu mpambanaji ni kuwa jasiri anayethubutu kupambana bila woga. Jasiri yuko tayari kujitoa mhanga kwa ajili ya watu wengine. Ujasiri ni hali ya asili lakini huweza kujengwa kupitia mafunzo na mazingira ya makuzi. Kwa hiyo, ujasiri siyo jambo la kawaida au jepesi katika maisha yoyote duniani. Hata hivyo, huwezi kuwa jasiri kamili kama hauna raslimali zinazokufanya uwe na huo ujasiri. Baadhi ya raslimali hizo ni akili inayochaji vema, roho ya kujiamini, maumbile bora ya mwili, uaminifu, juhudi, maarifa, ubunifu, stadi na mbinu bora za kimapambano. Raslimali nyingine ni nguvu za rohoni na mwilini na uchumi thabiti ili kusaidia kujenga himaya imara, usalama wa uhakika, ulinzi imara, pia watu waaminifu na makini walio upande wako.

Jamii nyingi zinazomdunisha mwanamke hazijawahi kumruhusu mwanamke kumiliki raslimali hizi. Kwani hawaamini wala kumwona mwanamke kama kiumbe mwenye uwezo na sifa za ujasiri, uthubutu na uvumilivu. Daima humwona kuwa kiumbe aliyejaa woga, wasiwasi, kukata tamaa na kutokujiamini kiasi cha kutoweza kuumba, kuratibu, kusimamia, kupigania wala kulinda chochote kwa ufanisi na mafanikio (Wazambi \& Komanya, 2019). Kinyume na mtazamo huu, mwandishi Edwin Semzaba amemuonesha mwanamke kuwa kiumbe mwenye hadhi-msingi hata wakati mwingine kumzidi mwanaume. Tunalithibitisha hili kupitia nukuu ifuatayo:

Angalia juu yako uone vibwengo vikija kutushambulia. Majaliwa aliangalia juи na kuona viumbe vyenye maumbo ya ajabu na ya kutisha vikija kama upepo. Aliogopa na kumuuliza bibi yake kwa wasiwasi "sasa bibi tutafanyaje?" "Usihofu. Dawa yao ndogo tu mjukuu wangu." Bibi alimwambia. Vile vibwengo idadi yao kama mia mbili vikawazunguka. Bibi akatoa pumzi chafu kwa nguvu, sauti yake kama mzinga. Basi vile vibwengo vyote vikakimbia na kutawanyika huku vimekamata pua zao. Harufu ya hewa chafu ilikuwa komesha. Majaliwa hakuweza kuipata kwa sababu ya kinga ya bibi yake. [...] Hivyo hawatarudi tena?" Majaliwa aliuliza. "Thubutu!" Bibi alimjibu. "Ile harufu ni kwa kuwa wewe haukuinusa haina mchezo. Nikitoa kwa binadamu watazimia kwa siku tatu" (uk. 25 - 26).

Bibi bila kupenda, aligeuza njia na kuanza kurudi walikotoka. Nyuki wale wakawa wanakuja kwa spidi kubwa. Ndipo bibi akasimama kwenye ule ungo kama kuhimiza waende mbio zaidi. Ungo uliongeza kasi na punde wakajiona wanaingia Tanga. Ilikuwa saa saba ya usiku. [...] Bibi akatua kwenye uwanja wa mpira wa Mkwakwani. "Hawana adabu vijana wale. Wameturudisha! Ngoja tupumzike 
kwa dakika chache halafu niwavae kikamilifu. Hawana adabu!" (uk. 51).

Nukuu hii inaonesha ujasiri alionao mwanamke katika kukabiliana na ukinzani anaokutana nao. Ujasiri huu hauji kama bahati nasibu, bali unatokana na uwezo alionao katika kukabiliana na vizingiti tofauti tofauti. Ujasiri wa mwanamke huyu unatokana na kujikomboa kwake kifikra na kiraslimali kulikomsaidia kuongoza mapambano dhidi ya mfumodume. Nukuu hii inaonesha wazi kwamba jitihada nyingi za mwanamke katika kupambania maisha zinapitia vikwazo vingi kutoka kwa mwanaume. Zamani, sifa ya ujasiri ilikuwa ikitazamwa kuwa ni sifa ya mwanaume pekee. Pale alipotokea mwanamke jasiri kupingana na mwanaume, jamii ilimuona ni nuksi na asiyefaa kuishi (Weathers, 1969/2010).

Mwanamke mara zote hutazamwa kama kiumbe wa nyumbani ambaye hawezi hata kusimama mbele ya mumewe na kusema neno wala kumiliki raslimali za asili ikiwemo ujasiri wa kuhodhi ardhi (Koda, 2000). Jamii nyingi zimeendelea kumtazama mwanaume kama kiongozi wa kila kitu. Kumbe hali haiko hivyo, mwanamke anaouwezo wa kutafuta, kumiliki, kulinda na kutenda kwa ujasiri kama wanavyotumainiwa wanaume.

\section{Mwanamke Kiumbe Mtumwa wa Asasi Zinazodunisha Hadhi-Msingi ya Mwanamke}

Kwa muda mrefu sasa, mwanamke ameonekana ndiye mwathirika mkuu wa dhuluma na ukatili unaoratibiwa chini ya mfumodume kupitia asasi mbalimbali za kijamii, kisiasa na kiuchumi (Wazambi \& Komanya, 2019). Miongoni mwa asasi hizo ni ndoa, dini na utamaduni. Utamaduni na dini zinaaminisha watu wanapofunga ndoa na kuyaanza maisha ya ndoa, hawapaswi kufikiria kutengana hata pale ndoa inapokuwa sawa na jehanamu kwa wanandoa. Mtazamo huu unapatikana katika mila za jamii nyingi pia katika mafundisho na imani za dini hususani dini ya Kikristo na Kiislamu. Kwa mfano kwa Wakristo, mafundisho husisitiza katika ndoa ya mke mmoja na isiyovunjika mpaka kifo kitakapowatenganisha wanandoa hao (Bible Society of Tanzania, 1997 - Mwanzo 2: 24, Mathayo 19: 3 - 9, Waefeso 5: 25 \& 33; Marko 10: 9, Mhubiri 4: 9, 1 Petro 3: 7, na Malaki 2:16).
Kwa upande wa dini ya Kiislamu, kama mwanaume ana uwezo wa kiuchumi na kiafya, basi anaruhusiwa kuongeza mwanamke mwingine mradi wasizidi wanne (4); maadamu mke / wake wameridhia. Hata hivyo, zipo taarifa zinazoonesha baadhi ya wanaume na viongozi wa dini hii kutokufuata kanuni na sheria za kidini kwa mujibu wa Kurani Tukufu na Sunna za Mtume Mohammed (S. A. W), (Mbyana, h.m). Hivyo, baadhi kama siyo wengi wa wanaume hufuata matakwa na matamanio yao katika suala la ndoa hizo. Pamoja na ukiukwaji huo, mara nyingi dini haingilii kati ili kumnusuru mwanamke, bali hubariki udhalimu huo kwa kumsihi mwanamke kuendelea kuvumilia na kuomba Mungu apate kumtetea (Karisa \& Mwinyifaki, 2017). Wazambi na Komanya (2019) wanaripoti kuwa, asasi ya ndoa, utamaduni na dini zimechangia sana baadhi ya wanawake kufanyiwa ukatili wa kijinsia wa kutisha. Matokeo yake, asasi hizi zimekuwa chanzo cha mateso na ukatili kwa mwanamke mnyonge. Edwin Semzaba ametambua ukubwa wa changamoto hii na kuipatia nafasi katika riwaya yake. Hapa mwandishi anasema:

Hapo kale babu yako alikuwa mume wangu. Alinitesa sana. Wakati huo tuliishi duniani. Babu alikuwa na mke mwingine, hivyo akawa hanijali mimi. Siku moja yeye na mkewe huyo wa pili walikula njama. Nilipokwenda kisimani kuchota maji wakati wa usiku walinitumbukiza, kisimani nikafariki. Nikaenda kuzimu ambako nilikuwa na bidii hata wengi wakanipenda. Naye babu yako alipokufa, akaja kuzimu, lakini kutokana na matendo yake mabaya alipokuwa duniani, akawekwa daraja la saba kwenye dhiki nyingi. [...] Huko kuzimu, nilitengeneza marimba ya sauti ishirini, nikawa nawatumbuiza watu siku za sikukuu. Marimba haya yalinipa umaarufu sana huko kuzimu [...]. Ili kuepukana na ghasia za babu yako na wenzake, niliamua nikuletee ile marimba ili uweze kushinda mashindano. [...] Lakini ghasia hazikuisha kule kuzimu. Ghasia hazikomi. Ndiyo maisha yetu. Babu yako aliamua kukupokonya marimba ili ampe kijukuu cha rafiki yake wa kuzimu. Kijukuu huyo ni ... malizia!" "Kongoti Nachienga" Majaliwa akasema (uk. 23 - 25).

Nukuu hii inakiri namna ndoa ya bibi ilivyokuwa na changamoto hasa baada ya babu kuoa mke wa pili. Mambo yaliharibika zaidi mara baada ya mke wa

76 This work is licensed under a Creative Commons Attribution 4.0 International License. 
pili kuungana na babu (mmewe) kumtesa bibi hadi kumuua. Kinachosikitisha zaidi ni kuona mwanamke akitumikia mfumodume ili kumuangamiza mwanamke mwenzake kisa asasi ya ndoa. Ukatili wa wanandoa hawa dhidi ya mwanandoa mwenzao ni uthibitisho wa namna asasi ya ndoa inavyotumiwa na mwanaume kumlaghai mwanamke mmoja ili kumdhibiti mwanamke mwingine. Hapa tunaona dhahiri ni kwa namna gani ndoa imekuwa kama asasi ya fitina na unyanyasaji wanawake. Mwandishi anaungana na wasanii wengine mathalani p'Bitek (1975), Mbogo (2002), Hanson (2008) na Mgimba (2017) kuonesha athari ya mfumodume kwa damu iliyotokana na mwanaume husika. Kwamba, ukatili wa mme au ndugu wa mme kwa mke, hauishii kwa mwanamke tu bali huhamishiwa kwa watoto na wajukuu wa mwanamke husika.

Ukatili huu umechangia sana ongezeko la ndoa kuvunjika katika nchi zilizoendelea kwa kuwa wengi wa wanawake wanatambua haki zao na sheria za nchi zinawalinda (Graviningen na wenzake, 2017). Hali hii ni tofauti katika nchi zinazoendelea ambako kuna sheria lakini wengi wa wanawake na wanajamii hawajakomboka kifikra. Hivyo, mwanamke na mtoto wanaendelea kuumia kila uchwao. Matukio ya ukatili wa kijinsia wenye kufanana na huu aliofanyiwa bibi na mjukuu wake katika ndoa bado yanaripotiwa hata hivi leo. Ripoti zinaonesha bado kuna matukio ya wanawake kupigwa hasa pale wanaume wanapokuwa wamelewa na kuanzisha vurugu bila kujali kuna watoto au wageni (Wazambi na Komanya, 2019). Ripoti zinaonesha kila mwaka kumekuwapo na ongezeko la mauaji na ukatili mwingine kwa mwanamke na mtoto kupitia asasi mbalimbali za kijamii, kitamaduni, kisiasa na kiuchumi.

Mbali ya mauaji, bado nyumba ndogo au minyukano ya wanaume kwa sababu anuai zimeendelea kuripotiwa kuwa chanzo cha mwanaume kutelekeza familia au nyumba kugeuka uwanja wa vita; wanawake na watoto kugeuka ombaomba, wakimbizi, wahanga wa ubakaji, ulawiti, dhiki, magonjwa, majeraha na ulemavu unaotokana na vipigo au matumizi ya silaha zinazodhuru mwili (International Organization for Migration, 2019), kutaja kwa uchache.
Hali hii ya wanaume kuwatesa wake zao kwa kushirikiana na baadhi ya wanawake ni tishio kwa ustawi endelevu wa mwanamke na jamii yote. Juhudi za haraka na za makusudi hazina budi kuchukuliwa kwa faida ya wote. Haya yote hayatokei kwa bahati mbaya bali ni sehemu ya mfumodume uliojichimbia kilindini mwa maisha ya mwanadamu (Levine, 1977). Ndiyo maana, pamoja na baadhi ya wanaharakati kutokukubaliana na mtazamo wake, mfeministi Gloria Steinem anaona ni heri mwanamke akajitenga kabisa na asasi ya ndoa, dini na mila. Kwani anaamini ukombozi wa kweli wa mwanamke hauwezi kufikiwa bila mwanamke huyo kujitenga na asasi hizo tatu (Nimavat, 2014).

Badru (2019) anatufunulia siri muhimu juu ya umwamba wa mfumodume. Kwamba, utamaduni wa wanaume kujiona wao ni kila kitu katika jamii, siyo tu kumewaumiza wanawake pekee. Bali umejenga mfumo uliowageuza hao wanaume kuwa watumwa wa wanawake pasipo wao kujua. Mafunzo na semi hutumika kuwatesa wanaume kwa kuhamasishwa kutafuta mali na ufahari ili kujenga uungumtu machoni mwa mwanamke - hata kama mwanaume husika hana uwezo huo. Ndiyo maana, zipo ripoti zinazoonesha kuwa hata wanaume baadhi wameanza kuonja shubiri ya kuteswa na wake au wapenzi wao wasiojitambua au waliojitambua lakini kutokana na athari za mfumo dume, wanawake hao wamegeuka wakatili sawasawa na wanaume wanaowakatili wanawake kila kukicha (Yakubu, 2014). Kwa hiyo, kupitia riwaya ya Marimba ya Majaliwa (2008), nadharia ya Ufeministi na wanataaluma tuliowarejelea, tunathibitisha kwamba, riwaya ya kingano inaweza kugusa mambo nyeti yakiwemo yale ya asasi ya ndoa na namna yanavyotumika kudunisha hadhimsingi ya mwanamke.

\section{Ukombozi wa Mwanamke na Maendeleo Yake Yanahitaji Ukombozi Binafsi wa Kifikra na Nyenzo Bora na Sahihi kwa Wakati Sahihi}

Maendeleo ya kweli na endelevu ya mwanamke yanahitaji ukombozi wa kweli wa kifikra kwa kutumia raslimali sahihi na bora (Hunter \& Sellers, 1998). Hooks (2000), Ward (2001), Mbogo (2002), Muhando (2007), Msuya (2012) na Mgimba (2017) wanaonekana kusisitiza umuhimu wa jamii kubadilika kimtazamo juu ya ukombozi wa 
mwanamke na maendeleo halisi ya jamii. Wanataaluma hawa wanaona kuwa, ukombozi wa kweli utapatikana endapo kuna umoja na mshikamano thabiti baina ya mwanamke na mwanaume. Naye Weathers (1969/2010) anaamini kuwa, ukombozi wa kweli wa mwanamke hauwezi kuletwa kwa kuwapo maneno matupu ya jukwaani. Bali, utaletwa kwa mwanamke mmoja mmoja kujikomboa kifikra na kuwa jasiri kupambana na vizingiti vinavyomkwamisha. Weathers anasisitiza sana katika umuhimu wa kujikomboa kifikra. Kwa sababu, kama mwanamke anayesema anapigania ukombozi wa mwanamke wakati yeye binafsi hajajikomboa kifikra na kinyenzo, atawezaje kuwakomboa wanawake wengine!

Riwaya ya Marimba ya Majaliwa haikubaki nyuma katika kulianika hadharani tatizo la wengi wa wanajamii wakiwemo wanawake kuendelea kuwa maamuma wa fikra tunduizi. Riwaya inaonesha bado kuna changamoto ya wanajamii kutokujikomboa kifikra kiasi cha kusababisha kukosekana kwa utangamano thabiti wa kumpigania mwanamke. Mwandishi anaichora jamii kama taasisi isiyo na msaada mkubwa katika harakati za kumkomboa mwanamke mnyonge. Wanajamii wengi wakiwemo wanawake, bado wangali katika kongwa la utumwa wa mfumodume pasipo wao wenyewe kujitambua kama ni watumwa. Kupitia riwaya hii, mwandishi analichora wazo hili kiubunifu kwa kumtumia mhusika bibi.

Bibi anaonekana kukabiliana vikali na kundi la mme wake (babu yake Majaliwa) na vibaraka wake. Mwanamke huyu anaonekana kunyimwa ushirikiano thabiti. Badala yake, wadhalimu wanajenga umoja na ushirikiano imara wenye lengo la kuendelea kumdunisha mwanamke mnyonge. Hata hivyo, kupitia kujikomboa kifikra na kinyenzo, mwanamke aliyejitambua na mwenye shime ya kujikwamua kutoka katika makucha kandamizi huibuka kidedea mbali ya kupitia misukosuko ya kila namna. Tunalithibitisha hili kupitia nukuu hii:

Hapo kale babu yako alikuwa mume wangu. Alinitesa sana. Wakati huo tuliishi duniani. Babu alikuwa na mke mwingine, hivyo akawa hanijali mimi. Siku moja yeye na mkewe huyo wa pili walikula njama. Nilipokwenda kisimani kuchota maji wakati wa usiku walinitumbukiza, kisimani nikafariki. Nikaenda kuzimu ambako nilikuwa na bidii hata wengi wakanipenda. [...] Saa saba za usiku bibi aliwasili. "Imebaki mikoa miwili tu uyapate marimba yako. Kazana na tumia akili yako." Bibi alimwambia Majaliwa huku wakipanda juu ya ufagio. [...] Hatimaye mtangazaji alipanda jukwaani na kutamka, "mshindi na bingwa wa Marimba nchini Tanzania ni Majaliwaaaaa!' Kulikuwa na vifijo vya ajabu. Askari walipata kazi ya ziada kuwaondoa watu Wasimsonge Majaliwa. Baada ya muda, walifanikiwa kuwaondoa watu isipokuwa mtu mmoja tu, mwanamke wa makamo bibi yake Majaliwa! Leo alikuwa amevaa tenge lake la Ngurdoto na kutokea katika umbo la mama yake Majaliwa. Alimnong'oneza Majaliwa sikioni. "Sikuweza kuja. Babu yako aliweka majeshi mengi ajabu alinishinda nguvu. Hata hivyo leo niko hapa. Hongera mjukuu wangu! Na marimba nakuachia kama zawadi yangu" (uk. 23, 205, 234).

Nukuu hizi zinaonesha mfano mzuri wa mwanamke aliyejikomboa kifikra na mwenye nyenzo thabiti, kujiamini, ujasiri na ari ya kuleta mabadiliko katika jamii. Bibi Majaliwa anaonekana kuwa mwanamke pekee katika riwaya ya Marimba ya Majaliwa, ambaye ni mfano mzuri wa kuigwa na wanawake wengine katika jamii. Bibi Majaliwa alijitahidi kwa hali na mali kupambana na maadui ili kuhakikisha mjukuu wake anapata marimba yake. Ingawa wakati fulani alionekana kuzidiwa nguvu, hakukata tamaa bali alifikiri upya namna ya kupambania haki na usawa katika jamii. Huyu anaweza kuwakomboa watu wanyonge hususani watoto na wanawake wengine wanaponyanyasika kwa sababu amejitambua kifikra na kinyenzo. Kwa upande wa pili, mke wa pili wa babu yake Majaliwa anaonekana kuwa mfano wa wanawake wasiojikomboa kifikra. Kwani alishindwa kumtetea mwanamke mwenzake badala yake aliungana na mume wake kumdhulumu bibi Majaliwa haki zake. Kupitia riwaya hii tumeziona hadhi-msingi nyingi za mwanamke na namna zinavyodhibitiwa kikamilifu na mfumodume.

Huu ni ushahidi wa dhati unaothibitisha athari ya jamii kuwa na kundi la watu wanyonge mathalani wanawake ambao hawajajikomboa kifikra. Matokeo yake, wanaendelea kutumika kuwadhibiti 
watu wasio na sauti katika jamii. Huu ni mfano mzuri unaolibeba vema wazo la Mary Ann Weathers juu ya ukombozi wa kweli wa mwanamke. Kwamba, kama mwanamke mmoja mmoja hatajikomboa kifikra, kamwe hawezi kuwakomboa wanawake wenzake. Badala yake ataendelea kuwa wakala wa tabaka tawala lenye kumdunisha yeye na wanawake wengine. Mtazamo huu ndio unaojitokeza pia katika tamthilia ya Nguzo Mama (2007) ya Penina Muhando. Kumbe basi, hata katika kazi bunilizi za kingano hususani riwaya mnaweza kuzaliwa kwa maudhui mazito yenye kuiandaa jamii ya watoto kutambua na kuchukia uovu wa mfumodume kwa mwanamke na mtoto. Tena ni ushahidi unaothibitisha kuwa riwaya ya kingano inabeba mafunzo mazito ya kupindua mfumodume katika siku za usoni kwa siha ya jamii nzima na mtu mmojammoja.

\section{HITIMISHO}

Makala haya yamethibitisha ya kuwa, kama riwaya ya kingano itatumika vizuri, basi itakuwa ni miongoni mwa majukwaa thabiti ya kuchochea na kuleta ukombozi wa kweli na endelevu wa mwanamke na jamii nzima ya watu kwa ustawi endelevu. Aidha, makala yamethibitisha ya kwamba, riwaya ya Marimba ya Majaliwa ni kazi bunilizi iliyotambua mchango wa malezi bora yenye lengo la kuleta mapinduzi sahihi kupitia watoto. Riwaya hii ilitungwa kwa ajili ya watoto. Kwa hiyo, kitendo cha mwandishi kuingiza nafasi ya mwanamke na mtoto katika jamii iliyojaa udhalimu kutokana na mfumodume, kutasaidia kuchochea ufahamu, ari na maono ya watoto kutamani kuibadilisha hali inayomdunisha mwanamke na mtoto. Kwa mantiki hii, mbali ya uhondo wa kifani, utalii na historia uliojazwa katika riwaya hii, riwaya hii ni chanzo kizuri cha mafundisho halisi ya hali na namna ya kuikomboa jamii kutoka katika makucha na mabavu ya mfumodume.

Riwaya hii inaitikia wito wa maandiko matakatifu hususani Biblia unaosema, "Mlee mtoto katika njia impasayo, naye hataiacha, hata atakapokuwa mzee (Mithali 22:6)." Nao wahenga walisema, "samaki mkunje angali mbichi." Kwa hiyo Edwin Semzaba ametumia kalamu na karatasi kuanza kumkunja mtoto mapema kabisa ili kutambua ukweli wa hali na ubaya wa propaganda chafu zinazomhusu mwanamke na asasi kandamizi dhidi ya ustawi wa mwanamke, mtoto na jamii nzima; pia njia za kuondoa udhalimu huo. Hali halisi huko uwandani inaonesha mwanamke na mtoto ndio wanaopata adha kubwa kutokana na mfumodume kuendelea kutokujali utu, haki, usawa, uwezo, utashi, nafasi, thamani na uhai wa kila binadamu kufurahia uumbaji wa Mungu.

\section{MAREJELEO}

Badru, Z. A. (2019). Mfumo Dume - Chanzo cha Ufungwa wa Mwanaume: Uhakiki wa Semi Kutoka Jamii za Kiafrika. Katika JULACE: Journal of University of Namibia Language Centre, Vol. 4 (1).

Bible Society of Tanzania. (1997). Maandiko Matakatifu ya Mungu yaitwayo BIBLIA. Nairobi: Africa Area Typesetting Unit.

Graviningen, K. na wenzake, (2017). Reported Reasons for Breakdown of Marriage and Cohabitation in Britain: Findings from the Third National Survey of Sexual Altitudes and lifestyle (Natsal-3). PLOS ONE 12 (3); eo174129. https;//doi.org/10.1371/journal.pone.0174129.

Hanson, B. J. (2008). Takadini (toleo la pili). Dar es Salaam: Methews Bookstore and Stationers.

Hardiman, R., Jackson, B. W., \& Griffin, P. (2010). Conceptual Foundations. Katika B. Adams, Castaneda, Hackman, Peters \& Zúnigana wenzake. (Wahariri), Readings for Diversity and Social Justice. New York, NY: Routledge.

Hewitt, N. A. (2010). Introduction. Katika N. A. Hewitt. (Mhariri). No Permanent Waves: Recasting Histories of U.S. Feminism. New Brunswick, NJ: Rutgers University Press.

Hooks, B. (2000). Feminism is for Everybody: Passionate Politics. Cambridge, MA: South End Press.

Hunter, A. G. \& Sellers, S. L. (1998). Feminist Attitudes among African American Women and Men. Katika Sage Journals. https://doi.org/10.1177/089124398012001005.

International Organization for Migration. (2019). World Migration Report 2020. 
https://publications.iom.int/system/files/pdf/wm r_2020.pdf.

Karisa, B. S. \& Mwinyifaki, A. (2017). 'Iktibasi' katika Tenzi za Mwanakupona na Al-Inkishafi. Katika Kioo cha Lugha, Vol. 11. https://journals.udsm.ac.tz/index.php/kcl/article/ view/786

Koda, B. (2000). The Gender Dimension of Land Rights in Tanzania: Case Study of Msindo Village, Same District. PhD Thesis (unpublished). University of Dar es salaam.

Levine, L. (1977). Black Culture and Black Consciousness: Afro - American Folk Thought From Slavery to Freedom. London: Oxford University Press.

Maggie, H. (1996). Feminist Literary Criticism in America and England. Katika Women's Writing: A Challenge to Theory. Sussex: Harvesters Press.

Masatu, F. na wenzake. (2020). Mwingilianomatini katika Tamthilia ya Utangamano Wetu Ushindi Wetu (2017) ya Anitha E. Mgimba. Katika East African Journal of Swahili Studies 2 (2), 128143. https://doi.org/10.37284/eajss.2.2.217.

Mbogo, E. (2002). Watoto wa Mama N'tilie. Dar es Salaam: HEKO Publishers.

Mbyana, H. H. (h.m). Ndoa ya Kiislam. https://www.alislam.org/swahili/Ndoa-yaKiislamu.pdf.

Mgimba, A. E. (2017). Utangamano Wetu Ushindi Wetu. Dar es Salaam: E \& CE Publishers.

Msuya, M. B. (2012). Usawiri wa Mhusika 'Bibi' katika Riwaya za Kiswahili: Mfano kutoka Riwaya za Marimba ya Majaliwa na Ngome ya Mianzi. Tasnifu ya Shahada ya Uzamili (haijachapishwa). Chuo Kikuu cha Dar es salaam.

Muhando, P. (2007). Nguzo Mama. Dar es Salaam: Macmillan Aidan Ltd.

Mulokozi, M. M. (2017). Taaluma ya Lugha na Fasihi - 2: Utangulizi wa Fasihi ya Kiswahili
Kozi za Fasihi Vyuoni na Vyuo Vikuu. Dar es salaam: KAUTTU.

Nimavat, S. (2014). Gloria Steinem - A Feminist Activist with a Difference (A Study of Her Major Views). Katika International Journal of Research and Analytical Reviews. http://ijrar.com/upload_issue/ijrar_issue_65.pdf.

p'Bitek, O. (1975). Wimbo wa Lawino: Ombolezo. Dar es Salaam: EAPH.

Pasque, P. A. (2011). Women of Color in Higher Education: Theoritical Perspectives. Katika G. Jean-Marie \& B. Llyod-Jones. (Wahariri), Women of Color in Higher Education: Turbulent Past, Promising Future, Vol. 9. Bingley, UK Emerald.

Pearson, R. (2000). Rethinking Gender Matters in Development. Katika Allen, T \& Thomas, A. (Wahariri). Poverty and Development into the $21^{\text {st }}$ Century. Oxford: Oxford University Press.

Rutherford, A., Marecek, J. \& Sheese, K. (2012). Psychology of Women and Gender. Katika Weiner Vol. $1 . \quad$ https://www. Researchgate.net/publication/312056770.

Semzaba, E. (2008). Marimba ya Majaliwa. Dar es salaam: E\&D Vision Publishing.

TUKI. (2005). Kamusi ya Kiswahili Sanifu (toleo la pili). Nairobi: Oxford University Press.

Ward, P. (2001). 'Women of Britain Say Go': Women's Patriotism in the First World War. Katika Twentieth Century British History, Vol. 12 (1). Doi: 10.1093/tcbh/12.1.23

Wazambi, F. \& Komanya, J. (2019). Ukatili wa Kingono: Tishio kwa Haki na Ustawi wa Mtoto Nchini Tanzania, Muhtasari wa Ripoti ya Haki za Binadamu Tanzania 2018: Tanzania Bara. Dares salaam: Kituo cha Sheria na Haki za Binadamu.

Weathers, M. A. (1969/2010). An Argument for Black Women's Liberation as a Revolutionary Force. http://scriptorium.lib.duke.edu/wlm/fungames2/argument.html.

$80 \mid$ This work is licensed under a Creative Commons Attribution 4.0 International License. 
Yakubu, A. M. (2014). Women as Their Own Worst Enemies: Rewrting the Stepmother and Motherin-Law Archetypes in Adimora-Ezeibo's Roses and Bullets. Katika IOSR Journal of Humanities and Social Sciences, Vol. 19 (1). http://www.iosrjournals.org/iosrjhss/papers/Vol19-issue1/Version11/C0191110915.pdf?id=8829.

81 This work is licensed under a Creative Commons Attribution 4.0 International License. 